\title{
Frontotemporal Lobar Degeneration: A Clinical Approach
}

\author{
Elissaios Karageorgiou, MD ${ }^{1,2}$ Bruce L. Miller, MD ${ }^{1}$ \\ ${ }^{1}$ Memory and Aging Center, Department of Neurology, University of \\ California San Francisco, San Francisco, California \\ 2 Neurological Institute of Athens, Athens, Greece \\ Address for correspondence Bruce L. Miller, MD, Department of \\ Neurology, Memory and Aging Center, University of California San \\ Francisco, 675 Nelson Rising Lane, Suite 190, San Francisco, CA 94158 \\ (e-mail: bmiller@memory.ucsf.edu).
}

Semin Neurol 2014;34:189-201.

\begin{abstract}
Keywords

- frontotemporal dementia

- primary progressive aphasia

- behavior

- language

- parkinsonism

- diagnosis

- treatment

In this review, the authors outline a clinical approach to frontotemporal lobar degeneration (FTLD), a term coined to describe a pathology associated with atrophy of the frontal and temporal lobes commonly seen with abnormal protein aggregates. It accounts for $\sim 10 \%$ of pathologically confirmed dementias. The three clinical syndromes associated with FTLD are jointly classified as frontotemporal dementia (FTD) and include behavioral variant frontotemporal dementia (bvFTD), nonfluent-agrammatic primary progressive aphasia (nfvPPA), and semantic variant PPA (svPPA; left: I-svPPA and right: rsVPPA). All syndromes have differential impairment in behavioral (bvFTD; r-svPPA), executive (bvFTD; nfvPPA), and language (nfvPPA; svPPA) functions early in the disease course. With all three there is relative sparing of short-term memory and visuospatial abilities early on, and with the two language syndromes, nfvPPA and svPPA, behavior is also intact. Symptoms are associated with specific atrophy patterns, lending unique imaging signatures to each syndrome (frontal: bvFTD and nfvPPA; temporal: svPPA). Common proteinopathies involve accumulation of tau, transactive response DNA binding protein 43, and fusion in sarcoma protein. Parkinsonism presents in all syndromes, especially cases with tau pathology and MAPT or GRN mutations. nfvPPA often has corticobasal degeneration or progressive supranuclear palsy as the underlying neuropathological substrate. bvFTD co-occurs with motor neuron disease in $\sim 15 \%$ of cases, and many such cases are due to C9Orf72 mutations. Other common genetic mutations in FTLD involve GRN and MAPT. Behavioral symptoms are best managed by selective serotonin reuptake inhibitors, while atypical antipsychotics should be used with caution given side effects. Promising etiologic treatments include anti-tau antibodies, antisense oligonucleotides, and progranulin enhancers.
\end{abstract}

Frontotemporal lobar degeneration (FTLD) is defined as a pathologic endophenotype characterized by atrophy of the frontal and temporal lobes leading to three clinical syndromes with partially overlapping microscopic pathology. These are jointly called frontotemporal dementia (FTD) and include the behavioral variant frontotemporal dementia (bvFTD) and two types of the primary progressive aphasias (PPA), ${ }^{1}$ the nonfluent-agrammatic (nfvPPA) and the semantic (svPPA). The three syndromes are associated with variable impairment in behavioral, executive, language, and even motor functions early in the disease course. Each has a unique atrophy pattern on neuroimaging. Commonly, there is accumulation of tau, transactive response DNA binding protein 43 (TDP-43), fusion in sarcoma protein (FUS), and p62 dipeptides. $^{2}$

In 2011, revised consensus criteria were created for both bvFTD and PPA to incorporate advances in imaging, pathology, and genetics, aiming to improve early diagnostic accuracy. 3,4
Issue Theme Atypical Parkinsonian Disorders; Guest Editors, Yvette Bordelon, MD, PhD, and Carlos PorteraCailliau, MD, PhD
Copyright (c) 2014 by Thieme Medical Publishers, Inc., 333 Seventh Avenue, New York, NY 10001, USA. Tel: +1(212) 584-4662. 
Clinical syndromes

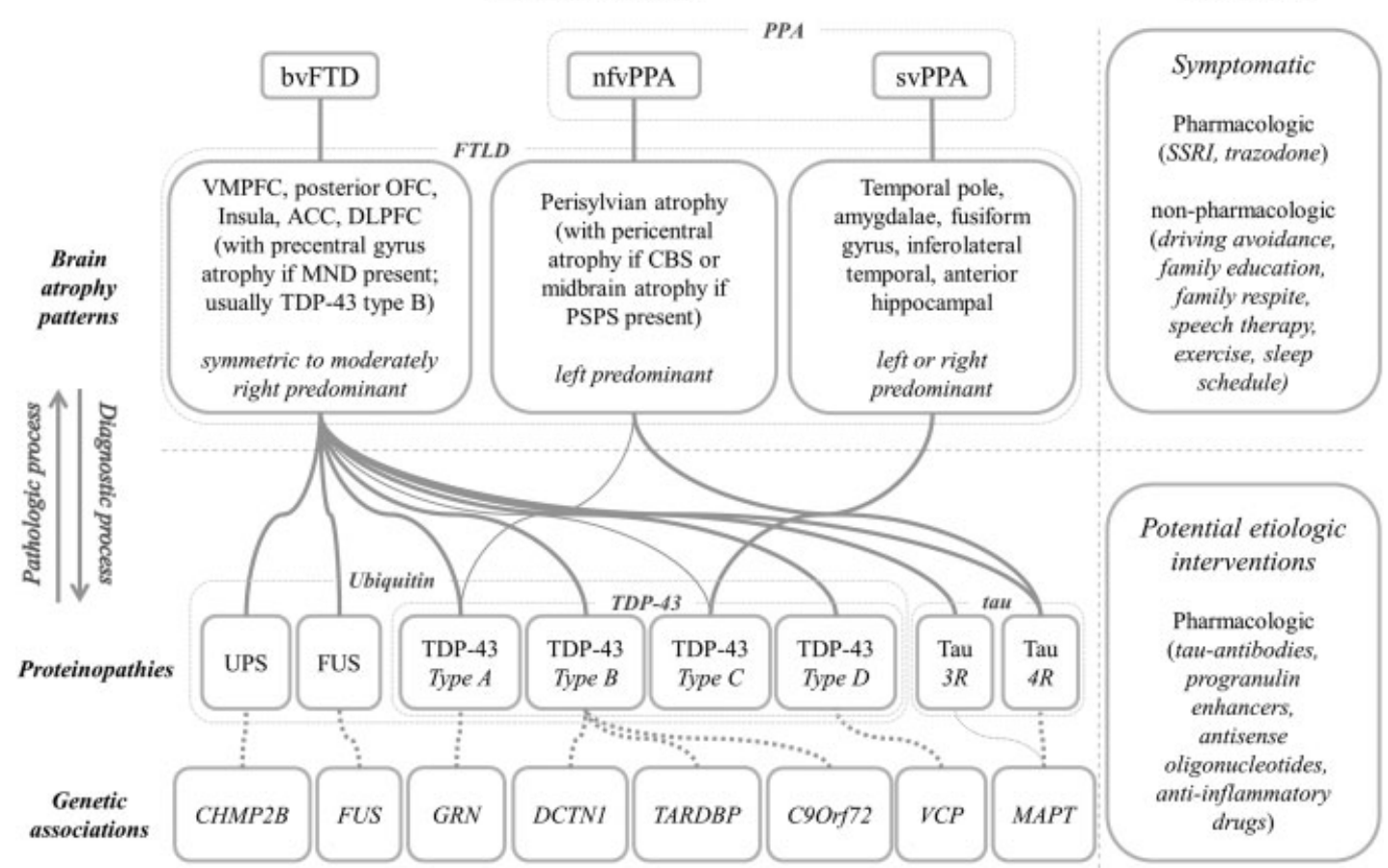

Fig. 1 Frontotemporal lobar degeneration (FTLD) phenotypes, endophenotypes, and therapeutic associations. Common atrophy patterns, pathologies, and genetic mutations are depicted. Syndromes correlate well to gross atrophy patterns; similarly, genetic mutations correlate to specific proteinopathies. Line weights represent relative associations. ACC, anterior cingulate cortex; bvFTD, behavioral variant frontotemporal dementia, CBS, corticobasal syndrome; CHMP2B, charged multivesicular body protein 2b; DCTN1, dynactin 1; DLPFC, dorsolateral prefrontal cortex; FUS, fusion in sarcoma; GRN, progranulin; MAPT, microtubule associated protein tau; MND, motor neuron disease; nfvPPA, nonfluentagrammatic PPA; OFC, orbitofrontal cortex; PPA, primary progressive aphasia; PSPS, progressive supranuclear palsy syndrome; sVPPA, sematic variant PPA; TARDBP, transactive response DNA binding protein gene; TDP-43, transactive response DNA binding protein 43; UPS, ubiquitin proteasome system; $V C P$, valosin containing protein; VMPFC, ventromedial prefrontal cortex.

Early and accurate diagnosis, however, is not straightforward in FTLD, given the pathological convergence associated with specific clinical syndromes and the syndromic divergence within pathologies (-Fig. 1). Even family members with a single genetic mutation are phenotypically heterogeneous.

\section{Historical Perspective and Epidemiology}

In 1892, Arnold Pick described patients with presenile dementia, aphasia, and lobar atrophy. ${ }^{5}$ This entity was subsequently referred to as Pick disease, and the characteristic inclusion bodies associated with this condition, identified by Alois Alzheimer in 1911, were named Pick bodies in Pick's honor. In 1957, Delay, Brion, and Escourolle and in 1974 Constantinidis, Richard, and Tissot delineated the clinical and anatomical differences between Alzheimer disease (AD) and Pick disease, emphasizing that atrophy in Pick disease was frontally predominant, while in AD more posterior. Their classification schemas recognized that there were prominent extrapyramidal syndromes associated with Pick disease and that only a minority of cases had classic Pick bodies. ${ }^{6,7}$ In 1982, Marsel Mesulam identified aphasia syndromes in patients with left-predominant hemispheric atrophy, ${ }^{8}$ collectively termed PPA (now including nfvPPA, SvPPA, and logopenic variant PPA [lvPPA]). ${ }^{1}$ Though Pick's first cases would currently be classified as svPPA of left-predominant atrophy (l-svPPA), in the past "Pick dementia" was considered synonymous to what is now called bvFTD. A rightpredominant atrophy svPPA (r-svPPA) also exists and presents with early behavioral deficits, whereas its syndromic convergence and pathologic homology to l-svPPA allows both syndromes to be classified as SvPPA (see below). Recent discoveries of specific proteinopathies (e.g., tau, TDP43, FUS) as well as genetic mutations (e.g., GRN, MAPT, C90rf72) has opened avenues for new therapeutic interventions. $^{9-16}$

Epidemiologically, FTLD incidence is three to four cases per 100,000 person-years, with an estimated 20,000 to 30,000 cases in the United States at a given moment. ${ }^{17}$ It is the third most common cause of degenerative dementia after AD and dementia with Lewy bodies, accounting for 5 to $10 \%$ of all pathologically confirmed cases. ${ }^{18}$ Additionally, it is the second most common presenile dementia in patients younger than 65 years old after AD. Tau-positive cases tend to exhibit older disease onset and slower progression than TDP-43 and FUS FTLD subtypes. ${ }^{19}$ - Table 1 contains epidemiologic features of FTLD subtypes, recognizing that diagnosis in most studies was based on pre-2011 diagnostic criteria. 
Table 1 Epidemiology of frontotemporal lobar degeneration (FTLD) $)^{19-22}$

\begin{tabular}{|l|l|l|l|l|}
\hline Clinical syndrome & $\begin{array}{l}\text { Percentage of } \\
\text { FTLD cases }\end{array}$ & $\begin{array}{l}\text { Range of male } \\
\text { percentage }\end{array}$ & $\begin{array}{l}\text { Mean age of } \\
\text { onset (range) }\end{array}$ & $\begin{array}{l}\text { Life expectancy in years } \\
\text { from symptom onset } \\
\text { (from diagnosis) }\end{array}$ \\
\hline bvFTD & $54-69$ & $53-70$ & $\begin{array}{l}58 \\
(47-82)\end{array}$ & $\begin{array}{l}\text { with MND 6 (1) } \\
\text { without MND 9 (5) }\end{array}$ \\
\hline nfvPPA & $14-35$ & $14-63$ & $\begin{array}{l}63 \\
(42-79)\end{array}$ & $9(4)$ \\
\hline r-svPPA & $6-10$ & $44-80$ & $\begin{array}{l}62 \\
(52-85)\end{array}$ & $12(5)$ \\
\hline I-svPPA & $9-12$ & $52-80$ & $\begin{array}{l}59 \\
(52-80)\end{array}$ & $12(5)$ \\
\hline
\end{tabular}

${ }^{a}$ No statistical difference.

${ }^{\mathrm{b}}$ Significantly shorter life expectancy only for bvFTD-MND cases.

\section{Clinical Diagnosis}

Frontotemporal lobar degeneration is caused by selective vulnerability of specific neuroanatomical networks. With bvFTD, nfvPPA, and svPPA degeneration starts within a specific hub and spreads across the respective network in a prion-like manner, conferring unique clinical characteristics at each stage of the disease. ${ }^{23-25}$ As such, the most important clinical information lies in the temporal evolution of symptoms, and by extension, their neuroanatomical representation, allowing the physician to create a mental map of brain atrophy progression. The diagnostic process aims to identify the phenotypic syndrome (i.e., bvFTD vs. nfvPPA vs. svPPA vs. other dementia or nondementia syndromes), and then predict the most likely proteinopathy and possible genetic mutation (-Figs. 1,2, and - Tables 2,3). ${ }^{31}$ This approach can provide a more accurate prognosis, and as molecule-specific therapies develop, more tailored treatment.

There are distinct differences between patients with rightversus left-sided disease. Right-predominant atrophy patients (bvFTD; r-svPPA) tend to be emotionally cold and distant, often disrupting family relationships, and present with behavioral disturbances that are often misinterpreted as psychiatric symptoms. Left-predominant atrophy patients mainly present with language impairments (-Table 2 ).

bvFTD is dominated by behavioral symptoms. Because early degeneration affects the paralimbic structures of the ventromedial prefrontal cortex (VMPFC), anterior cingulate cortex (ACC), and anterior insula, early symptoms involve social disinhibition, lack of motivation (apathy), and loss of empathy. ${ }^{31,34}$ Often, family members believe the patient has lost interest in the family, is depressed, or suffers from a psychiatric disorder. Patients are often distractible and it is not uncommon for them to lose their jobs. Symptoms of disinhibition may range from inappropriate (e.g., hugging people in the street) to antisocial (e.g., commenting on peoples' weight). Lack of empathy is striking, and patients may ignore acute health issues of their spouses. As selective degeneration spreads to the temporal lobes, particularly the right, mental rigidity and unique eating habits start to emerge (e.g., eating only single-colored food). Some patients may develop cravings for carbohydrate-rich food such as sweets and chips. Compulsive behaviors can range from simple repetitive movements (e.g., tapping, coughing) to more complex compulsions (e.g., hoarding, collecting, cleaning, eating specific foods at specific times). As the dorsolateral prefrontal cortex (DLPFC) degenerates, executive abilities falter, with working memory impairment, difficulty with set-shifting and generation of ideas or alterations in attention. ${ }^{36}$ Usually, patients have poor insight into their deficits, distort their history, and admit to having bvFTD as a matter-of-fact based on others' reports, rather than appreciating that something is amiss. This may relate to noso-adiaphoria (anosodiaphoria) rather than noso-agnosia (anosognosia). ${ }^{37}$

A slowly progressive bvFTD exists, termed "phenocopy" by Chris Kipps and John Hodges, which differs from the classic form due to decades-long progression and male predominance. ${ }^{38}$ It is indistinguishable from the classic form based on simple diagnostic criteria, although measures of executive and functional impairment tend to be less severe in the phenocopy cases and atrophy may be mild, or even absent. Some of these patients are primarily psychiatric, although C90rf72 mutations may also be responsible for the syndrome. ${ }^{39}$

One in seven bvFTD patients develop $M N D,{ }^{22}$ which has a similar phenotype to sporadic MND, although often lower limb muscles seem to be spared early on. Because bv FTD-MND has strong pathological associations with TDP-43 type B and C9Orf72 (and some other) mutations (-Table 3 ), it is often approached separately from bvFTD without MND. There is evidence of a C90rf72 mutation founder effect from 6,300 years ago in the Western world, making it the most common genetic cause of bvFTD-MND and accounting for about a third of familial cases, but these C9Orf72 appear to be rare in south and eastern Asia. ${ }^{13,14,44}$ C90rf72 mutations are large hexanucleotide repeat expansions (GGGGCC) in the intron region of chromosome 9, which leads to RNA nuclear accumulation and suppression of gene expression. The disease phenotype does not seem to depend on repeat length and there is only preliminary evidence that longer repeat sizes, specifically in the cerebellum, have a negative impact on survival. ${ }^{45}$ 

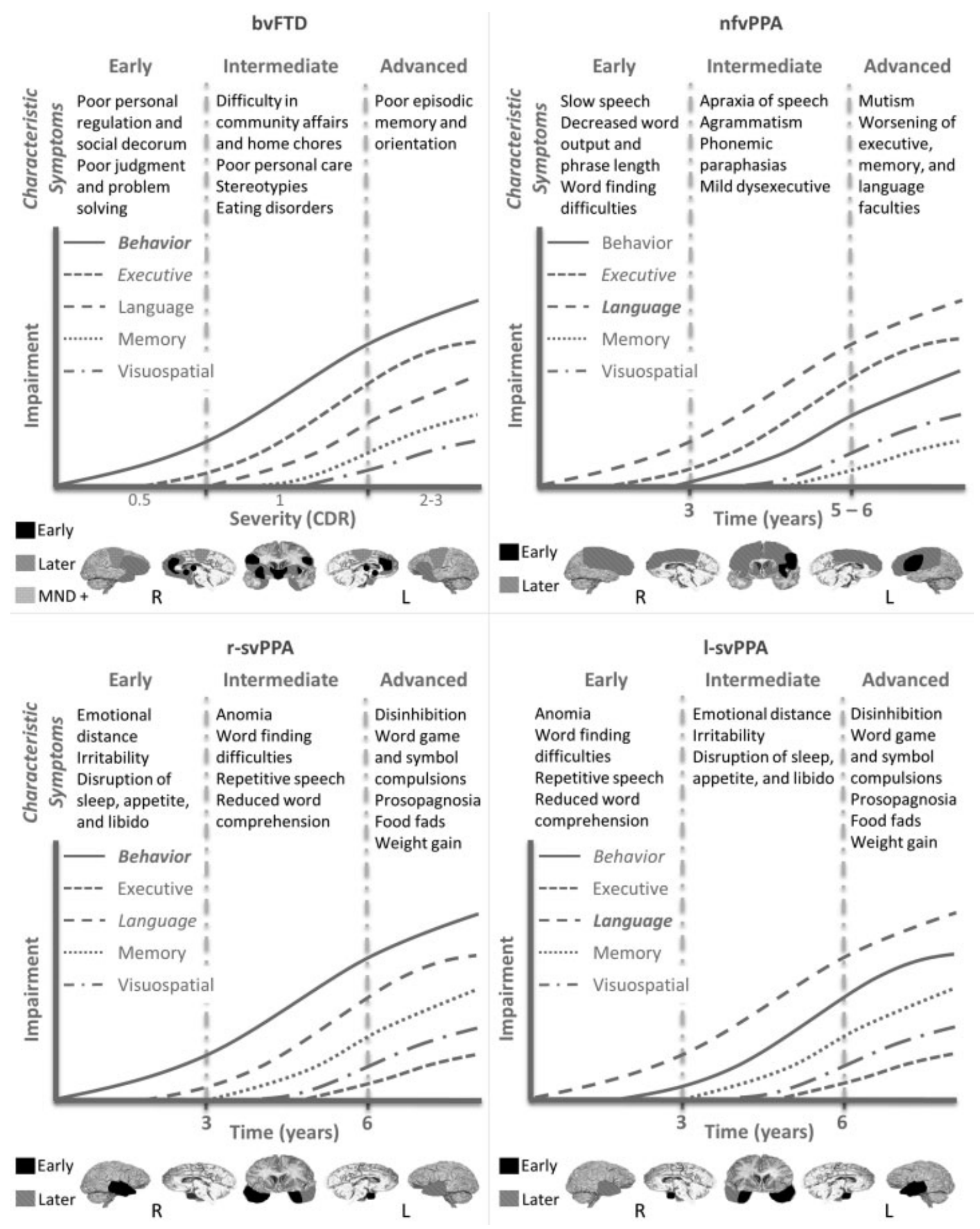

Fig. 2 Syndrome natural history in frontotemporal lobar degeneration. ${ }^{26-30}$ Common cognitive, behavioral, and atrophic patterns with disease progression; see text for details. Graphs depict relative qualitative symptom severity with disease progression. Speed of disease progression in bvFTD is more variable than other syndromes, with Clinical Dementia Rating (CDR) Scale scores ranging from 0.5 to 3 at 6 years from symptom onset.

$n f v P P A$ is the prototypical syndrome with impairments in language structure and praxis. Characteristic deficits include nonfluent output, agrammatism, and apraxia of speech (AOS). ${ }^{1,3}$ Patients understand the meaning of individual words or objects, but have trouble with more complex sentences. The neuroanatomical network affected by degeneration includes the dominant frontal operculum, its con- nections to the supplementary motor area (SMA) through the frontal aslant tract, the premotor area, and the insular cortex. ${ }^{28,46}$ Thus, early symptoms are slowness of speech, word-finding difficulties, and decreased word output and phrase length. Apraxia of speech (i.e., an articulation planning deficit) emerges as a disconnection between the frontal operculum and the SMA, associated with aslant tract 


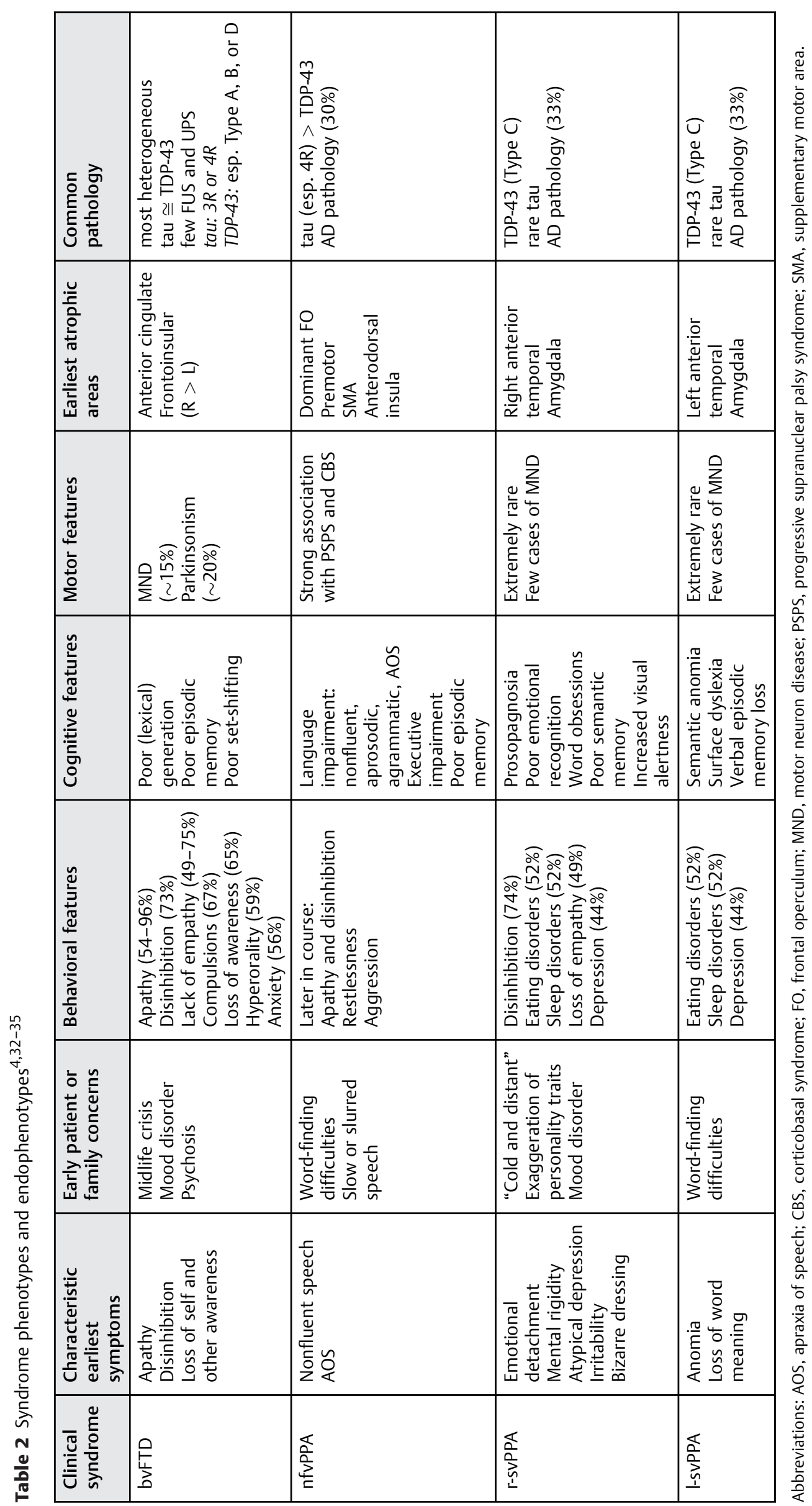


Table 3 Clinicopathological associations $2,9-16,40-43$

\begin{tabular}{|c|c|}
\hline Clinical syndrome & Characteristic clinicopathological associations \\
\hline bvFTD & $\begin{array}{l}\text { tau } \\
\text { Parkinsonism common (including CBS); “parietal” symptoms (e.g., acalculia) more } \\
\text { common than in ubiquitin cases } \\
\text { Symmetric frontal atrophy involving temporal lobes; more prominent striatal atrophy and } \\
\text { white matter abnormalities than ubiquitin cases } \\
\text { MAPT mutations (Chromosome 17) } \\
\text { TDP-43 type A (see below) } \\
\text { TDP-43 type B } \\
\text { Associated with bvFTD-MND; parkinsonism (rarely CBS) } \\
\text { Mildly asymmetric frontal atrophy and parietal, pulvinar and cerebellar atrophy } \\
\text { C9Orf72 mutations (Chromosome 9; Baltic ancestry; most common known genetic cause) } \\
\text { Less common genes: TARDBP (Italian/French ancestry; parkinsonism and MND), DCTN1 } \\
\text { (Perry syndrome) } \\
\text { TDP-43 Type D } \\
\text { bvFTD ( } \pm \text { MND), IBM and Paget disease of the bone; parkinsonism uncommon } \\
\text { VCP gene (Chromosome 9) } \\
\text { FUS } \\
\text { Younger onset (30s to 40s); associated with bvFTD-MND; psychotic features (up to 36\%) } \\
\text { FUS mutations (Chromosome 16) } \\
\text { UPS } \\
\text { CHMP2B mutations (Chromosome 3; Denmark) } \\
\text { Other genes related to TDP-43 pathology } \\
\text { UBQLN2 (MND, X-linked, mean onset 30s to 40s), OPTN (MND), hnRNPA1, and A2/B1 (IBM } \\
\text { and Paget disease) }\end{array}$ \\
\hline nfvPPA & $\begin{array}{l}\text { TDP-43 type A } \\
\text { Parkinsonism frequent (including CBS) } \\
\text { Asymmetric atrophy of dorsolateral frontoparietal lobes and basal ganglia } \\
\text { GRN mutations (Chromosome 17) } \\
\text { tau } \\
\text { Strongly associated with AOS } \\
\text { Usually CBD or PSP }\end{array}$ \\
\hline I-svPPA & $\begin{array}{l}\text { TDP-43 Type C } \\
\text { Movement disorders uncommon; coexistence of autoimmune diseases and } \\
\text { left-handedness } \\
\text { Left-predominant anterior temporal atrophy } \\
\text { Almost exclusive pathology; rarely genetic }\end{array}$ \\
\hline r-svPPA & $\begin{array}{l}\text { TDP-43 Type C } \\
\text { Movement disorders uncommon; coexistence of autoimmune diseases and } \\
\text { left-handedness } \\
\text { Right-predominant anterior temporal atrophy } \\
\text { Almost exclusive pathology; rarely genetic }\end{array}$ \\
\hline
\end{tabular}

Abbreviations: AOS, apraxia of speech; CBD, corticobasal degeneration; CBS, corticobasal syndrome; CHMP2B, charged multivesicular body protein 2b; DCTN1, dynactin 1; FUS, fusion in sarcoma; GRN, progranulin; hnRNPA, heterogeneous nuclear ribonucleoprotein; IBM, inclusion body myositis; MND, motor neuron disease; OPTN, optineurin; PSP, progressive supranuclear palsy; TARDBP, TAR DNA-binding protein; UBQLN2, ubiquilin 2; UPS, ubiquitin proteasome system; $V C P$, valosin containing protein.

degeneration. ${ }^{46}$ In contrast, agrammatism, which in addition to simplified phrases is defined by omission of function words and inflections, develops with progressive atrophy of the left frontal operculum and DLPFC, but also of the insula, anterosuperior temporal cortex, as well as white matter degeneration of the dominant cingulum and corpus callosum. ${ }^{47}$ Emergence of phonemic paraphasias (e.g., phoneme transpositions, additions, omissions) relates to progressive atrophy of the insula, anterior cingulate, premotor cortex, and SMA. $^{47}$ In contrast to bvFTD, nfvPPA patients often become aware of their deficits prior to others and maintain a proper social decorum. As the disease moves into the contralateral frontal regions, some nfvPPA patients eventually develop behavioral disturbances. Finally, nfvPPA often coincides with corticobasal syndrome (CBS) or progressive supranuclear palsy syndrome (PSPS), in which a 4-repeat tauopathy is probable, although CBS may also result from TDP-43 Type A pathology with or without GRN mutations (-Table 3).

Both SvPPA syndromes have semantic knowledge deficits with intact speech fluency, but differ in that early symptoms in 1svPPA pertain to lexical meaning loss, whereas in r-svPPA to loss of emotional meaning and knowledge about faces. ${ }^{48}$ Symptoms correlate to early atrophy of the anterior temporal pole, which serves as a hub for semantic knowledge and from which degeneration spreads. ${ }^{25,26,28}$ Disease spreads to frontal areas once the uncinate fasciculus becomes affected, highlighting its 
role in semantic processing, ${ }^{46}$ while there is accompanying atrophy of the insula and anterior hippocampus. ${ }^{26,28}$

Early features of l-svPPA include word-finding difficulties, especially of nouns rather than verbs. Gradually, patients substitute specific words with superordinate categories (e.g., animal for cat), and eventually most nouns are called things. At later stages, loss of word meaning becomes very pronounced and patients have trouble recognizing what is shown to them and its general purpose. In contrast, early features of r-svPPA are behavioral, in keeping with an underlying right-predominant atrophy, while language problems present later in its course. ${ }^{20,48} \mathrm{r}$-svPPA manifests with early emotional detachment, lack of empathy, and diagnosis is often delayed because symptoms are misinterpreted as psychiatric or worsening of chronic personality traits. Some patients' symptoms begin with impairment recognizing familiar faces, and evolve into a severe deficit in facial perception.

At intermediate svPPA stages, degeneration spreads to the opposite hemisphere and the two svPPA subtypes merge at the syndromic and atrophic level (-Fig. 2). In clinic, patients may show surface dyslexia, in which they incorrectly read irregularly-written low-frequency words (e.g., yacht). svPPA patients develop an interest for visually appealing objects, which may express itself as compulsions or artistic creativity. De novo creativity is a fascinating feature in FTLD, especially lsvPPA, which may emerge a few years prior to the onset of disabling symptoms, and is probably caused by abolishment of interhemispheric inhibition.

\section{Parkinsonism in Frontotemporal Lobar Degeneration}

Approximately one-fifth of bvFTD patients have parkinsonism on their first clinic visit. ${ }^{35}$ Parkinsonian features are more common in bvFTD and nfvPPA patients, often those with tau pathology, MAPT and GRN mutations, and at later disease stages, whereas its presence does not affect survival (-Tables 2 and 3). ${ }^{22,35}$ Most bvFTD cases have an akineticrigid form (60\%) and the rest (40\%) are tremor-predominant. Movement disorders rarely accompany svPPA.

Corticobasal syndrome and PSPS are often considered as clinical diagnoses when parkinsonism is present early in FTLD. Unlike classic Parkinson disease, where rigidity, tremor, and bradykinesia dominate the early phases, PSPS presents with axial rigidity, relative sparing of the arms, and lack of tremor. Presence of early falls and a supranuclear gaze palsy is typical for PSPS. Corticobasal syndrome is characterized by apraxia (especially of the feet), alien limb phenomenon, inattention, dystonia, and myoclonus. Cortical symptoms (e.g., aphasia) overlap with those observed in bvFTD and nfvPPA. ${ }^{49,50}$ Corticobasal syndrome and PSPS are designed to predict 4-repeat tauopathies (i.e., corticobasal degeneration [CBD] and progressive supranuclear palsy [PSP], respectively). Although clinicopathological association is high for PSPS, CBS criteria have not been highly predictive and up to $50 \%$ of cases have alternative pathologies (e.g., AD [23\%] and TDP-43 $[13 \%])^{51}$ As a result, CBS criteria were recently revised, though their clinical utility and diagnostic accuracy remains to be seen. ${ }^{49}$
Parkinsonism in FTLD can also be due to specific genetic mutations. Two such genes are MAPT and GRN, which are 1.7 $\mathrm{Mb}$ apart on chromosome 17. GRN mutation deficits caused by progranulin haploinsufficiency have a mean age at onset of 59 years; MAPT mutations tend to present at an earlier age with a mean age at onset of 49 years. Life expectancy from the time of diagnosis is approximately 7 years for both. Shared signs of parkinsonism are rigidity and bradykinesia without a resting tremor. Furthermore, GRN mutation patients have asymmetric parkinsonism earlier in their course, and often display CBS, whereas MAPT mutation patients have a more symmetric akinetic-rigid parkinsonism and less typically exhibit CBS. On MRI, GRN mutation patients often show asymmetric atrophy that extends to the parietal lobes, and white matter signal abnormalities are common. In MAPT mutation cases, atrophy is more symmetric and parietal atrophy is not typically present. ${ }^{52}$ Another gene associated with parkinsonism and often MND is TARDBP, ${ }^{53}$ a rare mutation that has been reported in patients of Italian-French ancestry. In addition to rigidity and bradykinesia, rest tremor is more prevalent than in other FTLD-related mutations. C90rf72 and FUS mutations are also associated with parkinsonism, but, more typically, MND dominates their motor symptoms.

An interesting, yet unique, parkinsonism association in FTLD is the amyotrophic lateral sclerosis-Parkinson-dementia complex (ALS-PDC) of Guam ${ }^{54}$ ALS-PDC is strongly familial, but no genetic or environmental cause has been verified, while its prevalence has gradually declined. Clinically, there is rigidity, bradykinesia, and a nondisabling action-induced tremor. Finally, linear pigment retinal epitheliopathy occurs in $56 \%$ of cases compared with $16 \%$ of controls.

\section{Additional Studies}

In addition to obtaining a history of present illness and performing a physical examination, which provide the most useful diagnostic information, workup for suspected FTLD should include neuropsychological testing and structural brain MRI. Neuropsychological testing allows confirmation of historically reported cognitive deficits. It may not be significantly abnormal in the early stages of bvFTD or r-svPPA because early symptoms are mostly behavioral. In nfvPPA and l-svPPA specific tests of language are required. Generally, bvFTD patients have deficits in executive control, svPPA patients have language difficulties, evident on confrontation naming, and nfvPPA patients perform poorly on fluent output, word generation, and understanding of complex syntax comprehension. ${ }^{3,36}$ Tests of social cognition focused around social perception and behavior are helpful and may emerge in FTD prior to the onset of changes in executive control. ${ }^{55}$

One cornerstone of the FTLD workup is structural brain MRI. As reflected in - Fig. 2 and -Tables $\mathbf{2}$ and 3, atrophy patterns vary between syndromes and even between genetic mutations within syndromes. ${ }^{28,31,40}$ Clinicians should look for these changes in MRI sequences themselves and should not rely solely on the radiologist's impression, as radiologists often fail to comment on atrophy patterns. Additionally, MRI helps rule out other causes of cognitive and behavioral 
Table 4 Criteria for the diagnosis of bvFTD, nfvPPA, and svPPA ${ }^{1,3,4}$

\begin{tabular}{|c|c|c|c|c|}
\hline Syndrome & Possible/clinical diagnosis & $\begin{array}{l}\text { Probable/imaging } \\
\text { supported diagnosis }^{b}\end{array}$ & $\begin{array}{l}\text { Definite/pathologically or } \\
\text { genetically proven } \\
\text { diagnosis }\end{array}$ & Exclusionary criteria \\
\hline bvFTD & $\begin{array}{l}\text { At least } 3 \text { of the following: } \\
\text { - Early behavioral } \\
\text { disinhibition } \\
\text { - Early apathy or inertia } \\
\text { - Early lack of empathy } \\
\text { or sympathy } \\
\text { - Early perseverations, } \\
\text { stereotypies or } \\
\text { compulsions } \\
\text { - Dietary habit changes } \\
\text { or hyperorality } \\
\text { - Executive- } \\
\text { predominant deficits } \\
\text { on neuropsychological } \\
\text { testing with relative } \\
\text { sparing of memory } \\
\text { and visuospatial skills }\end{array}$ & $\begin{array}{l}\text { All of the following: } \\
\text { - Meets possible criteria } \\
\text { - Significant decline per } \\
\text { informant, or CDR, or } \\
\text { FAQ } \\
\text { - Imaging consistent } \\
\text { with bvFTD } \\
\text { (frontal and/or } \\
\text { anterotemporal) }\end{array}$ & $\begin{array}{l}\text { All of the following: } \\
\text { - Meets possible OR } \\
\text { probable criteria } \\
\text { - Histopathological } \\
\text { evidence of FTLD and/ } \\
\text { or presence of known } \\
\text { pathogenic mutation }\end{array}$ & $\begin{array}{l}\text { - Deficits are not better } \\
\text { explained by alterna- } \\
\text { tive diagnosis (degen- } \\
\text { erative, nondegenera- } \\
\text { tive, or psychiatric) }\end{array}$ \\
\hline nfvPPA $^{d}$ & $\begin{array}{l}\text { At least one of the } \\
\text { following: } \\
\text { • Agrammatism } \\
\text { Effortful, halting } \\
\text { speech with } \\
\text { inconsistent sound } \\
\text { errors (AOS) } \\
\text { At least two of the } \\
\text { following: } \\
\text { - Impaired } \\
\text { comprehension of } \\
\text { syntactically complex } \\
\text { sentences } \\
\text { - Spared single-word } \\
\text { comprehension } \\
\text { - Spared object } \\
\text { knowledge }\end{array}$ & $\begin{array}{l}\text { All of the following: } \\
\text { - Meets possible/clinical } \\
\text { criteria } \\
\text { - Imaging consistent } \\
\text { with nfvPPA } \\
\text { (left posterior } \\
\text { frontoinsular) }\end{array}$ & $\begin{array}{l}\text { All of the following: } \\
\text { - Meets possible OR } \\
\text { probable criteria } \\
\text { - Histopathological } \\
\text { evidence of specific } \\
\text { pathologyc and/or } \\
\text { presence of known } \\
\text { pathogenic mutation }\end{array}$ & $\begin{array}{l}\text { - Deficits are not } \\
\text { better explained } \\
\text { by alternative } \\
\text { diagnosis } \\
\text { (nondegenerative, } \\
\text { or psychiatric) } \\
\text { - Prominent initial } \\
\text { deficits are not } \\
\text { memory, } \\
\text { visuospatial, or } \\
\text { behavioral }\end{array}$ \\
\hline SVPPA $^{d}$ & $\begin{array}{l}\text { All of the following: } \\
\text { - Impaired confrontation } \\
\text { naming } \\
\text { - Impaired single-word } \\
\text { comprehension } \\
\text { At least } 3 \text { of the following: } \\
\text { - Impaired object } \\
\text { knowledge } \\
\text { - Surface dyslexia or } \\
\text { dysgraphia } \\
\text { - Spared repetition } \\
\text { - Spared grammar and } \\
\text { motor speech } \\
\text { production }\end{array}$ & $\begin{array}{l}\text { All of the following: } \\
\text { - Meets possible/clinical } \\
\text { criteria } \\
\text { - Imaging consistent } \\
\text { with svPPA (anterior } \\
\text { temporal lobe) }\end{array}$ & $\begin{array}{l}\text { All of the following: } \\
\text { - Meets possible OR } \\
\text { probable criteria } \\
\text { - Histopathological } \\
\text { evidence of specific } \\
\text { pathologyc and/or } \\
\text { presence of known } \\
\text { pathogenic mutation }\end{array}$ & $\begin{array}{l}\text { - Deficits are not } \\
\text { better explained } \\
\text { by alternative } \\
\text { diagnosis } \\
\text { (nondegenerative, } \\
\text { or psychiatric) } \\
\text { - Prominent initial } \\
\text { deficits are not } \\
\text { memory, } \\
\text { visuospatial, or } \\
\text { behavioral }\end{array}$ \\
\hline
\end{tabular}

Abbreviations: AOS, apraxia of speech; CDR, Clinical Dementia Rating Scale; FAQ, Functional Activities Questionnaire; FTLD, frontotemporal lobar degeneration; PET, positron emission tomography; SPECT, single-photon emission computed tomography.

${ }^{\mathrm{a}}$ Approximately within the first 3 years from symptom onset.

bImaging refers to structural magnetic resonance imaging atrophy, PET hypometabolism, or SPECT hypoperfusion.

'Specific pathology in 2011 PPA (primary progressive aphasia) criteria may be tau, TDP-43, Alzheimer disease, or other proteinopathy.

${ }^{d}$ Both nfvPPA and svPPA must satisfy PPA criteria by Mesulam ${ }^{1}$ with language impairment being the most prominent, disabling, and earliest symptom.

impairment, such as tumors, vascular disease, prion, and paraneoplastic disorders; hence the need for sequences such as diffusion weighted imaging, fluid attenuated inversion recovery, and gradient echo. ${ }^{28,31}$ In contrast to MRI, there are no characteristic changes on electroencephalography, other than mild frontal slowing.
Functional resting state imaging in FTLD, such as metabolism-associated positron emission tomography (PET), singlephoton emission computed tomography, and functional MRI, highlights impairments to vulnerable brain networks associated with behavioral and cognitive deficits (i.e., frontal and anterior temporal lobes). ${ }^{35}$ A conceptually similar approach 


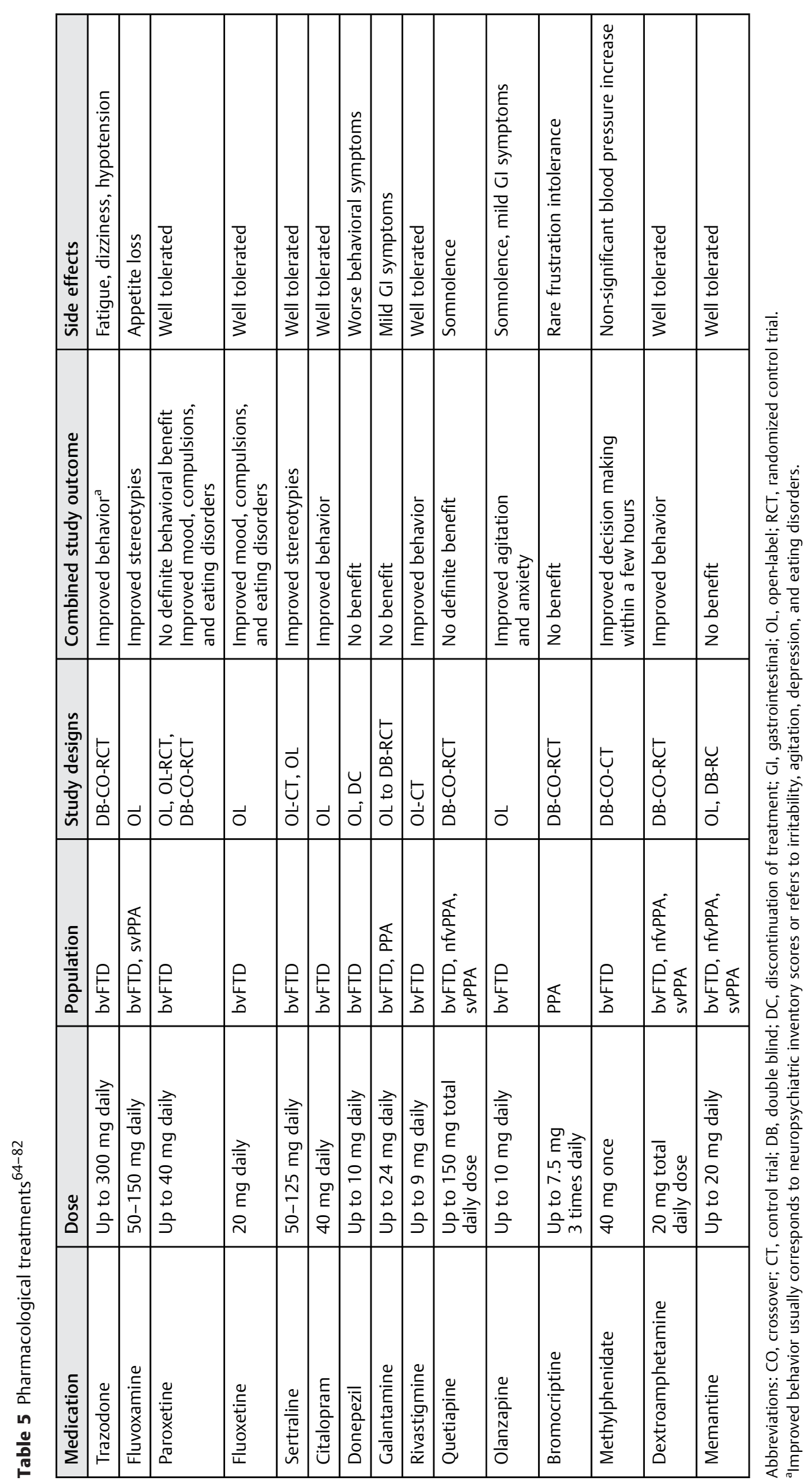


uses diffusion tensor imaging, which represents the structural integrity of white matter tracts connecting brain hubs. White matter tracts are affected early in the disease process, even in presymptomatic FTLD mutation carriers, and may provide even better diagnostic accuracy than volumetric MRI. ${ }^{56,57}$

Fluid biomarkers, such as blood and cerebrospinal fluid (CSF) have been extensively studied in FTLD. Testing for genetic mutations is useful if an autosomal dominant mutation is suspected (-Table 3). A risk factor for FTLD-tau, especially CBD and PSP, is histone 1 haplotype. ${ }^{58}$ In contrast, minor TMEM106B allele homozygosity protects GRN and C90rf72 mutation carriers. ${ }^{59,60}$ The best studied CSF biomarker is the tau: $A \beta_{1-42}$ ratio, which is significantly lower in FTLD than $\mathrm{AD}$ patients. ${ }^{61}$

Molecular PET is useful to test for the presence of amyloid pathology. Current guidelines recommend its use by a dementia expert ( 1 ) in patients younger than 65 years old, (2) in persistent or progressive unexplained mild cognitive impairment, and (3) in atypical or mixed-dementia presentations. $^{62}$ Thus, it is helpful in differentiating AD from bvFTD, or lvPPA from nfvPPA, or to identify dual pathology. Tau imaging will soon be available to search for tau-positive forms of FTLD. ${ }^{63}$ Currently, there is no TDP-43 or FUS PET.

\section{Diagnostic Criteria}

Frontotemporal lobar degeneration diagnostic criteria were revised in 2011 for both bvFTD and PPA, aiming to improve diagnostic accuracy (- Table 4)..$^{3,4}$ Nonetheless, there is still room for criteria improvement because diagnostic accuracy and interrater reliability is imperfect. In time, it is likely that criteria will incorporate molecular PET, improving direct syndrome-to-pathology diagnostic associations (-Fig. 1), while in parallel addressing multiple copathologies (e.g., AD and FTLD).

\section{Treatment}

- Table 5 lists symptomatic treatments tested in FTD trials. Prior etiologic treatments have either proven toxic or nonefficacious. ${ }^{83}$ For more details on FTD therapies, see also the review in the current issue by Tsai and Boxer, Clinical Trials: Past, Current, and Future for Atypical Parkinsonian Syndromes. In general, selective serotonin reuptake inhibitors are mildly beneficial for compulsions and eating disorders. Dopaminergic medications have no definite behavioral benefit. Recent trials do not support the use of memantine, and cholinesterase inhibitors seem to worsen behavior. Atypical antipsychotics should be used with caution only in cases of severe agitation given their extrapyramidal side effects. Levodopa may be considered in parkinsonism, especially where tau pathology or MAPT mutations are suspected, but a sustained response is rarely present.

A promising etiologic therapy focuses on halting tau spread using anti-tau antibodies, which in animal models decrease protein accumulation and improve behavior. ${ }^{84} \mathrm{An}-$ tisense oligonucleotides are being studied in C90rf72 mutations. ${ }^{85}$ There is a single report of steroid treatment improving symptoms in SvPPA, highlighting its association
Table 6 Foundations and Support Groups

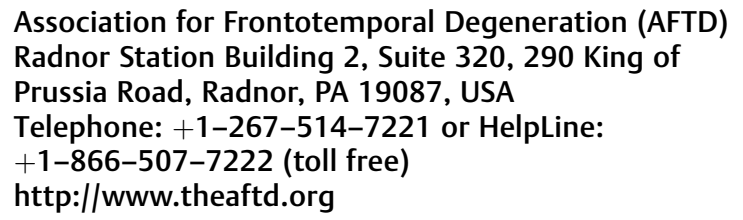

Consortium for Frontotemporal Dementia Research The Bluefield Project to Cure Frontotemporal Dementia 1650 Owens Street, Room 205, San Francisco, CA 94158, USA

http://www.bluefieldproject.org/contact-us

FRONTIER Frontotemporal Dementia Research Group NeuRA, PO Box 1165, Randwick NSW 2031, Australia

Telephone: $+61-2-9399-1000$

https://www.neura.edu.au/contact-us

Tau Consortium

http://tauconsortium.org

The Foundation for PSP | CBD and Related Brain Diseases (CurePSP)

30 E. Padonia Road, Suite 201, Timonium,

MD 21093, USA

Telephone: $+1-410-785-7004$ or $+1-800-457-4777$

(toll free)

http://www.psp.org

National Institute of Neurological Disorders and Stroke (NINDS)

Patient Recruitment and Public Liaison Office:

$+1-800-411-1222$ (toll free)

http://www.ninds.nih.gov/disorders/picks/picks.htm

The Frontotemporal Dementia Support Group

(United Kingdom)

The National Brain Appeal, Box 123, Queen Sq,

London, WC1N 3BG, UK

Regional contact information: http://www.ftdsg.org/

Regional_contacts

Frontotemporal Dementia Caregiver Support Center http://ftdsupport.com

Family Caregiver Alliance

785 Market Street, Suite 750, San Francisco,

CA 94103, USA

Telephone: $+1-415-434-3388$ or $+1-800-445-8106$

http://www.caregiver.org

Neil L. Radin Caregivers Relief Foundation

4404 Aberdeen Lane Blackwood, Blackwood,

N] 08012, USA

Telephone: +1-215-205-3162

FTD Support Forum

http://ftdsupportforum.com

to autoimmunity. ${ }^{86}$ Finally, treatments that raise progranulin levels are in development for GRN mutations. ${ }^{83}$

Nonpharmacological management of FTLD is as important as are pharmacological therapies. Family education and respite, a regular sleep schedule, social worker involvement, driving evaluation, exercise, and speech therapy can improve patients' and families' quality of life. Thus, a multidisciplinary dementia clinic is the optimal setting for management of FTLD. - Table 6 contains information on foundations and support groups for FTLD. 


\section{Acknowledgments}

Funding support for this work was provided through a Hellman Family Fellowship, the Consortium for Frontotemporal Dementia Research, the Tau Consortium, and NIH grant NIA-PPG P01-AG1972403.

\section{References}

1 Mesulam MM. Primary progressive aphasia. Ann Neurol 2001; 49(4):425-432

2 Rademakers R, Neumann M, Mackenzie IR. Advances in understanding the molecular basis of frontotemporal dementia. Nat Rev Neurol 2012;8(8):423-434

3 Gorno-Tempini ML, Hillis AE, Weintraub S, et al. Classification of primary progressive aphasia and its variants. Neurology 2011; 76(11):1006-1014

4 Rascovsky K, Hodges JR, Knopman D, et al. Sensitivity of revised diagnostic criteria for the behavioural variant of frontotemporal dementia. Brain 2011;134(Pt 9):2456-2477

5 Pick A. Über die Beziehungen der senilen Hirnatrophie zur Aphasie. Prager Medicinische Wochenschrift 1892;17:165-167

6 Constantinidis J, Richard J, Tissot R. Pick's disease. Histological and clinical correlations. Eur Neurol 1974;11(4):208-217

7 Delay J, Brion S, Escourolle R. Limites et conception actuelle de la maladie de Pick; son diagnostic différentiel. Ann Med Psychol (Paris) 1957;115(4):609-634

8 Mesulam MM. Slowly progressive aphasia without generalized dementia. Ann Neurol 1982;11(6):592-598

9 Hutton M, Lendon CL, Rizzu P, et al. Association of missense and 5'splice-site mutations in tau with the inherited dementia FTDP-17. Nature 1998;393(6686):702-705

10 Spillantini MG, Murrell JR, Goedert M, Farlow MR, Klug A, Ghetti B. Mutation in the tau gene in familial multiple system tauopathy with presenile dementia. Proc Natl Acad Sci U S A 1998;95(13):7737-7741

11 Baker M, Mackenzie IR, Pickering-Brown SM, et al. Mutations in progranulin cause tau-negative frontotemporal dementia linked to chromosome 17. Nature 2006;442(7105):916-919

12 Cruts M, Gijselinck I, van der Zee J, et al. Null mutations in progranulin cause ubiquitin-positive frontotemporal dementia linked to chromosome 17q21. Nature 2006;442(7105):920-924

13 DeJesus-Hernandez M, Mackenzie IR, Boeve BF, et al. Expanded GGGGCC hexanucleotide repeat in noncoding region of C9ORF72 causes chromosome 9p-linked FTD and ALS. Neuron 2011;72(2): 245-256

14 Renton AE, Majounie E, Waite A, et al; ITALSGEN Consortium. A hexanucleotide repeat expansion in C9ORF72 is the cause of chromosome 9p21-linked ALS-FTD. Neuron 2011;72(2): 257-268

15 Arai T, Hasegawa M, Akiyama H, et al. TDP-43 is a component of ubiquitin-positive tau-negative inclusions in frontotemporal lobar degeneration and amyotrophic lateral sclerosis. Biochem Biophys Res Commun 2006;351(3):602-611

16 Neumann M, Sampathu DM, Kwong LK, et al. Ubiquitinated TDP-43 in frontotemporal lobar degeneration and amyotrophic lateral sclerosis. Science 2006;314(5796):130-133

17 Knopman DS, Roberts RO. Estimating the number of persons with frontotemporal lobar degeneration in the US population. J Mol Neurosci 2011;45(3):330-335

18 Snowden JS, Neary D, Mann DM. Frontotemporal dementia. Br J Psychiatry 2002;180:140-143

19 Hodges JR, Davies R, Xuereb J, Kril J, Halliday G. Survival in frontotemporal dementia. Neurology 2003;61(3):349-354

20 Ioannidis P, Konstantinopoulou E, Maiovis P, Karacostas D. The frontotemporal dementias in a tertiary referral center: classification and demographic characteristics in a series of 232 cases. J Neurol Sci 2012;318(1-2):171-173
21 Johnson JK, Diehl J, Mendez MF, et al. Frontotemporal lobar degeneration: demographic characteristics of 353 patients. Arch Neurol 2005;62(6):925-930

22 Roberson ED, Hesse JH, Rose KD, et al. Frontotemporal dementia progresses to death faster than Alzheimer disease. Neurology 2005;65(5):719-725

23 Seeley WW, Crawford RK, Zhou J, Miller BL, Greicius MD. Neurodegenerative diseases target large-scale human brain networks. Neuron 2009;62(1):42-52

24 Kfoury N, Holmes BB, Jiang H, Holtzman DM, Diamond MI. Transcellular propagation of tau aggregation by fibrillar species. J Biol Chem 2012;287(23):19440-19451

25 Guo CC, Gorno-Tempini ML, Gesierich B, et al. Anterior temporal lobe degeneration produces widespread network-driven dysfunction. Brain 2013;136(Pt 10):2979-2991

26 Brambati SM, Rankin KP, Narvid J, et al. Atrophy progression in semantic dementia with asymmetric temporal involvement: a tensor-based morphometry study. Neurobiol Aging 2009;30(1):103-111

27 Seeley WW, Bauer AM, Miller BL, et al. The natural history of temporal variant frontotemporal dementia. Neurology 2005; 64(8):1384-1390

28 Gorno-Tempini ML, Dronkers NF, Rankin KP, et al. Cognition and anatomy in three variants of primary progressive aphasia. Ann Neurol 2004;55(3):335-346

$29 \mathrm{Lu}$ PH, Mendez MF, Lee GJ, et al. Patterns of brain atrophy in clinical variants of frontotemporal lobar degeneration. Dement Geriatr Cogn Disord 2013;35(1-2):34-50

30 Seeley WW, Crawford R, Rascovsky K, et al. Frontal paralimbic network atrophy in very mild behavioral variant frontotemporal dementia. Arch Neurol 2008;65(2):249-255

31 Rosen HJ, Gorno-Tempini ML, Goldman WP, et al. Patterns of brain atrophy in frontotemporal dementia and semantic dementia. Neurology 2002;58(2):198-208

32 Knibb JA, Xuereb JH, Patterson K, Hodges JR. Clinical and pathological characterization of progressive aphasia. Ann Neurol 2006; 59(1):156-165

33 Marczinski CA, Davidson W, Kertesz A. A longitudinal study of behavior in frontotemporal dementia and primary progressive aphasia. Cogn Behav Neurol 2004;17(4):185-190

34 Liu W, Miller BL, Kramer JH, et al. Behavioral disorders in the frontal and temporal variants of frontotemporal dementia. Neurology 2004;62(5):742-748

35 Le Ber I, Guedj E, Gabelle A, et al; French research network on FTD/ FTD-MND. Demographic, neurological and behavioural characteristics and brain perfusion SPECT in frontal variant of frontotemporal dementia. Brain 2006;129(Pt 11):3051-3065

36 Kramer JH, Jurik J, Sha SJ, et al. Distinctive neuropsychological patterns in frontotemporal dementia, semantic dementia, and Alzheimer disease. Cogn Behav Neurol 2003;16(4):211-218

37 Mendez MF, Shapira JS. Loss of emotional insight in behavioral variant frontotemporal dementia or "frontal anosodiaphoria". Conscious Cogn 2011;20(4):1690-1696

38 Kipps CM, Hodges JR, Hornberger M. Nonprogressive behavioural frontotemporal dementia: recent developments and clinical implications of the 'bvFTD phenocopy syndrome'. Curr Opin Neurol 2010;23(6):628-632

39 Khan BK, Yokoyama JS, Takada LT, et al. Atypical, slowly progressive behavioural variant frontotemporal dementia associated with C9ORF72 hexanucleotide expansion. J Neurol Neurosurg Psychiatry 2012;83(4):358-364

40 Rohrer JD, Warren JD. Phenotypic signatures of genetic frontotemporal dementia. Curr Opin Neurol 2011;24(6):542-549

41 Janssens J, Van Broeckhoven C. Pathological mechanisms underlying TDP-43 driven neurodegeneration in FTLD-ALS spectrum disorders. Hum Mol Genet 2013;22(R1):R77-R87

42 Miller ZA, Rankin KP, Graff-Radford NR, et al. TDP-43 frontotemporal lobar degeneration and autoimmune disease. J Neurol Neurosurg Psychiatry 2013;84(9):956-962 
43 Miller ZA, Mandelli ML, Rankin KP, et al. Handedness and language learning disability differentially distribute in progressive aphasia variants. Brain 2013;136(Pt 11):3461-3473

44 Smith BN, Newhouse S, Shatunov A, et al. The C9ORF72 expansion mutation is a common cause of ALS+/-FTD in Europe and has a single founder. Eur J Hum Genet 2013;21(1):102-108

45 van Blitterswijk M, Dejesus-Hernandez M, Niemantsverdriet E, et al. Association between repeat sizes and clinical and pathological characteristics in carriers of C9ORF72 repeat expansions (Xpansize-72): a cross-sectional cohort study. Lancet Neurol 2013;12(10):978-988

46 Catani M, Mesulam MM, Jakobsen E, et al. A novel frontal pathway underlies verbal fluency in primary progressive aphasia. Brain 2013;136(Pt 8):2619-2628

47 Ash S, Evans E, O'Shea J, et al. Differentiating primary progressive aphasias in a brief sample of connected speech. Neurology 2013; 81(4):329-336

48 Thompson SA, Patterson K, Hodges JR. Left/right asymmetry of atrophy in semantic dementia: behavioral-cognitive implications. Neurology 2003;61(9):1196-1203

49 Armstrong MJ, Litvan I, Lang AE, et al. Criteria for the diagnosis of corticobasal degeneration. Neurology 2013;80(5):496-503

50 Litvan I, Agid Y, Calne D, et al. Clinical research criteria for the diagnosis of progressive supranuclear palsy (Steele-RichardsonOlszewski syndrome): report of the NINDS-SPSP international workshop. Neurology 1996;47(1):1-9

51 Lee SE, Rabinovici GD, Mayo MC, et al. Clinicopathological correlations in corticobasal degeneration. Ann Neurol 2011;70(2): 327-340

52 Boeve BF, Hutton M. Refining frontotemporal dementia with parkinsonism linked to chromosome 17: introducing FTDP-17 (MAPT) and FTDP-17 (PGRN). Arch Neurol 2008;65(4):460-464

53 Borroni B, Archetti S, Del Bo R, et al. TARDBP mutations in frontotemporal lobar degeneration: frequency, clinical features, and disease course. Rejuvenation Res 2010;13(5):509-517

54 Lee SE. Guam dementia syndrome revisited in 2011. Curr Opin Neurol 2011;24(6):517-524

55 Elamin M, Pender N, Hardiman O, Abrahams S. Social cognition in neurodegenerative disorders: a systematic review. J Neurol Neurosurg Psychiatry 2012;83(11):1071-1079

56 Santillo AF, Mårtensson J, Lindberg $\mathrm{O}$, et al. Diffusion tensor tractography versus volumetric imaging in the diagnosis of behavioral variant frontotemporal dementia. PLoS ONE 2013;8(7):e66932

57 Dopper EG, Rombouts SA, Jiskoot LC, et al. Structural and functional brain connectivity in presymptomatic familial frontotemporal dementia. Neurology 2013;80(9):814-823

58 Houlden H, Baker M, Morris HR, et al. Corticobasal degeneration and progressive supranuclear palsy share a common tau haplotype. Neurology 2001;56(12):1702-1706

59 Finch N, Carrasquillo MM, Baker M, et al. TMEM106B regulates progranulin levels and the penetrance of FTLD in GRN mutation carriers. Neurology 2011;76(5):467-474

60 van Blitterswijk M, Mullen B, Nicholson AM, et al. TMEM106B protects C9ORF72 expansion carriers against frontotemporal dementia. Acta Neuropathol 2014;127(3):397-406[Epub ahead of print]

61 Bian H, Van Swieten JC, Leight S, et al. CSF biomarkers in frontotemporal lobar degeneration with known pathology. Neurology 2008;70(19 Pt 2):1827-1835

62 Johnson KA, Minoshima S, Bohnen NI, et al. Appropriate use criteria for amyloid PET: A report of the amyloid imaging task force, the society of nuclear medicine and molecular imaging, and the Alzheimer's association. J Nucl Med 2013;54(3): 476-490

63 Maruyama M, Shimada H, Suhara T, et al. Imaging of tau pathology in a tauopathy mouse model and in Alzheimer patients compared to normal controls. Neuron 2013;79(6):1094-1108
64 Herrmann N, Black SE, Chow T, Cappell J, Tang-Wai DF, Lanctôt KL. Serotonergic function and treatment of behavioral and psychological symptoms of frontotemporal dementia. Am J Geriatr Psychiatry 2012;20(9):789-797

65 Swartz JR, Miller BL, Lesser IM, Darby AL. Frontotemporal dementia: treatment response to serotonin selective reuptake inhibitors. J Clin Psychiatry 1997;58(5):212-216

66 Moretti R, Torre P, Antonello RM, Cazzato G, Bava A. Effects of selegiline on fronto-temporal dementia: a neuropsychological evaluation. Int J Geriatr Psychiatry 2002;17(4):391-392

67 Moretti R, Torre P, Antonello RM, Cazzato G, Griggio S, Bava A Olanzapine as a treatment of neuropsychiatric disorders of Alzheimer's disease and other dementias: a 24-month follow-up of 68 patients. Am J Alzheimers Dis Other Demen 2003;18(4): 205-214

68 Moretti R, Torre P, Antonello RM, Cazzato G, Bava A. Frontotemporal dementia: paroxetine as a possible treatment of behavior symptoms. A randomized, controlled, open 14-month study. Eur Neurol 2003;49(1):13-19

69 Moretti R, Torre P, Antonello RM, Cattaruzza T, Cazzato G, Bava A. Rivastigmine in frontotemporal dementia: an open-label study. Drugs Aging 2004;21(14):931-937

70 Mendez MF, Shapira JS, Miller BL. Stereotypical movements and frontotemporal dementia. Mov Disord 2005;20(6):742-745

71 Ikeda M, Shigenobu K, Fukuhara R, et al. Efficacy of fluvoxamine as a treatment for behavioral symptoms in frontotemporal lobar degeneration patients. Dement Geriatr Cogn Disord 2004;17(3): 117-121

72 Lebert F, Stekke W, Hasenbroekx C, Pasquier F. Frontotemporal dementia: a randomised, controlled trial with trazodone. Dement Geriatr Cogn Disord 2004;17(4):355-359

73 Mendez MF, Shapira JS, McMurtray A, Licht E. Preliminary findings: behavioral worsening on donepezil in patients with frontotemporal dementia. Am J Geriatr Psychiatry 2007;15(1):84-87

74 Kimura T, Takamatsu J. Pilot study of pharmacological treatment for frontotemporal dementia: risk of donepezil treatment for behavioral and psychological symptoms. Geriatr Gerontol Int 2013;13(2):506-507

75 Kertesz A, Morlog D, Light M, et al. Galantamine in frontotemporal dementia and primary progressive aphasia. Dement Geriatr Cogn Disord 2008;25(2):178-185

76 Huey ED, Garcia C, Wassermann EM, Tierney MC, Grafman J. Stimulant treatment of frontotemporal dementia in 8 patients. J Clin Psychiatry 2008;69(12):1981-1982

77 Reed DA, Johnson NA, Thompson C, Weintraub S, Mesulam MM. A clinical trial of bromocriptine for treatment of primary progressive aphasia. Ann Neurol 2004;56(5):750

78 Rahman S, Robbins TW, Hodges JR, et al. Methylphenidate ('Ritalin') can ameliorate abnormal risk-taking behavior in the frontal variant of frontotemporal dementia. Neuropsychopharmacology 2006;31(3):651-658

79 Diehl-Schmid J, Förstl H, Perneczky R, Pohl C, Kurz A. A 6-month, open-label study of memantine in patients with frontotemporal dementia. Int J Geriatr Psychiatry 2008;23(7):754-759

80 Boxer AL, Knopman DS, Kaufer DI, et al. Memantine in patients with frontotemporal lobar degeneration: a multicentre, randomised, double-blind, placebo-controlled trial. Lancet Neurol 2013; 12(2):149-156

81 Boxer AL, Lipton AM, Womack K, et al. An open-label study of memantine treatment in 3 subtypes of frontotemporal lobar degeneration. Alzheimer Dis Assoc Disord 2009;23(3):211-217

82 Vercelletto M, Boutoleau-Bretonnière C, Volteau C, et al; French research network on frontotemporal dementia. Memantine in behavioral variant frontotemporal dementia: negative results. J Alzheimers Dis 2011;23(4):749-759

83 Boxer AL, Gold M, Huey E, et al. Frontotemporal degeneration, the next therapeutic frontier: molecules and animal models for 
frontotemporal degeneration drug development. Alzheimers Dement 2013;9(2):176-188

84 Yanamandra $\mathrm{K}$, Kfoury $\mathrm{N}$, Jiang $\mathrm{H}$, et al. Anti-tau antibodies that block tau aggregate seeding in vitro markedly decrease pathology and improve cognition in vivo. Neuron 2013;80(2): 402-414
85 Lagier-Tourenne C, Baughn M, Rigo F, et al. Targeted degradation of sense and antisense C9orf72 RNA foci as therapy for ALS and frontotemporal degeneration. Proc Natl Acad Sci U S A 2013; 110(47):E4530-E4539

86 Decker DA, Heilman KM. Steroid treatment of primary progressive aphasia. Arch Neurol 2008;65(11):1533-1535 